\title{
Prevalencia de patologías pulmonares relacionadas con el asbesto
}

\section{Prevalence of pulmonary diseases related to asbestos}

LILIANA FERNÁNDEZ T., MD.(1)

En el comienzo del siglo xxi el mundo aun se enfrenta a conductas medioambientales y ocupacionales graves bien reconocidas, como es el uso de las fibras de asbesto en varios procesos y productos industriales. En la mayoría de los países se ha restringido su empleo y se han aumentado las medidas de prevención laboral.

El término asbesto se refiere a dos minerales diferentes: uno es el serpentine del cual el asbesto blanco crisolito es el más común y el otro es el asbesto amfibol eque incluye el asbesto azul crocidolita y el asbesto café amosita. El crisolito es el único tipo usado comercialmente hoy en día. En la literatura mundial se ha demostrado que tiene poca biopersistencia en el pulmón y al parecer no produce respuesta patológica en términos de inhalación a corto o largo término ni en el pulmón ni en la cavidad pleural (1). Estudios en trabajadores del cemento/crisolito han demostrado que bajo condiciones controladas el uso del crisolito puede ser seguro (2).

De todas maneras las enfermedades relacionadas con la exposición al asbesto siguen siendo la causa más común de morbilidad y mortalidad en trabajadores expuestos. El asbesto es la causa ocupacional más importante de cáncer del tracto respiratorio y la única de mesotelioma, además es una causa sustancial y probablemente subdiagnosticada de fibrosis pulmonar y enfermedad pleural debilitante (3). A pesar de sus bien conocidos efectos tóxicos, en general probablemente 125 millones de personas en el mundo están actualmente expuestas al asbesto en su trabajo (4).

En este número de la revista se presenta un artículo original relacionado con la investigación en un ambiente laboral de productos de fibrocemento que maneja fibras de asbesto crisolito bajo condiciones de higiene industrial controladas. Se estudió la prevalencia de las patologías relacionadas con la exposición a las fibras de crisolito en el aire en la población activa en un tiempo determinado, la historia de exposición acumulada a fibras, la antigüedad en la empresa y la historia de tabaquismo. Es un estudio de corte transversal que incluyó la evaluación radiológica de los sujetos acorde con la clasificación internacional de la
${ }^{(1)}$ Medicina Interna, Neumología, Neumología Intervencionista. Línea de Investigación Biomédica en Tórax, Fundación Valle del Lili. Profesora Clínica Asociada, Facultad de Ciencias de la Salud, Universidad Icesi. Cali, Colombia. Correspondencia: Liliana Fernández T., correos electrónicos: lfernandez@fcvl.org lilianafernandeztrujllo@gmail.com Recibido: 03/12/16. Aceptado: 15/12/16. 
Organización internacional del trabajo de radiografías de neumoconiosis según grupos de edad.

Los resultados concuerdan con los encontrados en la literatura mundial y se hace énfasis en el mantenimiento estricto de las medidas de seguridad laboral, la vigilancia epidemiológica continua para la identificación de casos índice precozmente y el seguimiento a largo plazo de la población expuesta activa e incluso la que ya no trabaja en el sitio de trabajo, que también puede tener enfermedad a largo plazo.

\section{Bibliografía}

1. Bernstein D. The health risk of chrysolite asbestos. Curr Opin Pulm Med. 2014;20:366-70.

2. Sichletidis L, Chloros D, Spyratos D, Haidich AB, Fourkiotou I, Kakoura M, et al. Mortality from occupational exposure to relatively pure chrysolite: a 39-year study. Respiration. 2009;78(1):63-8.

3. Barber CM, Wiggans RE, Young C, Fishwick D. UK asbestos imports and mortality due to idiopathic pulmonary fibrosis. Occup Med. 2016;66:106-11.

4. WHO. Asbestos: elimination of asbestos-related diseases. 2016. Disponible en: http://www.who.int/mediacentre/factsheets/fs343/en/ [Acceso 12 Sep 2016]. 